\title{
Efficacy and tolerability of the combination of nano-liposomal irinotecan and 5-fluorouracil/leucovorin in advanced pancreatic adenocarcinoma: post-approval clinic experience
}

\author{
Anup Kasi ${ }^{1 \#}$, Timothy McGinnis ${ }^{1 \#}$, Gurudatta Naik², Shivani Handa ${ }^{3}$, Grant Williams ${ }^{2}$, Ravi Paluri ${ }^{2}$ \\ ${ }^{1}$ Department of Medicine, Division of Medical Oncology, Kansas University Medical Center, Kansas City, KS, USA; ${ }^{2}$ Department of Medicine, \\ Division of Medical Oncology, University of Alabama at Birmingham, Birmingham, AL, USA; ${ }^{3}$ Department of Internal Medicine, Icahn School of \\ Medicine at Mount Sinai West \& Morningside, NY, NY, USA \\ Contributions: (I) Conception and design: A Kasi, R Paluri; (II) Administrative support: None; (III) Provision of study materials or patients: A Kasi, \\ T McGinnis, R Paluri; (IV) Collection and assembly of data: A Kasi, T McGinnis, R Paluri; (V) Data analysis and interpretation: G Naik, A Kasi, S \\ Handa, R Paluri; (VI) Manuscript writing and review: All authors; (VII) Final approval of manuscript: All authors. \\ "These authors contributed equally to this work. \\ Correspondence to: Anup Kasi. Department of Medicine, Division of Medical Oncology, Kansas University Medical Center, 2650 Shawnee Mission \\ Pkwy, Kansas City, KS 66205, USA. Email: akasi@kumc.edu.
}

Background: Nano-liposomal irinotecan (nal-IRI) plus 5-fluorouracil/leucovorin (5-FU/LV) is the regimen of choice in the $2^{\text {nd }}$ line setting for advanced pancreatic adenocarcinoma (PAC). However, realworld data is limited. Our objectives were to elicit the real-word effectiveness and safety of this combination as an advanced line of therapy in pancreatic cancer patients and analyze the impact of prior lines of therapy on survival outcomes with this regimen.

Methods: We conducted a retrospective cohort study of 58 patients with locally advanced unresectable or metastatic PAC, who were treated with at least one dose of nal-IRI + 5-FU/LV following cancer progression on prior therapies between August 2015 and December 2018 at the Kansas University Medical Center (KUMC) and University of Alabama at Birmingham (UAB).

Results: Median OS was 5.4 (range, 4.2-7) months. Disease control rate (DCR) was highest (84\%) for patients given nal-IRI $+5-\mathrm{FU} / \mathrm{LV}$ as $2^{\text {nd }}$ line agent after progression on a $1^{\text {st }}$ line gemcitabine-based regimen. However, no significant survival difference was observed between those given nal-IRI $+5-\mathrm{FU} / \mathrm{LV}$ after $1^{\text {st }}$ line or beyond the $2^{\text {nd }}$ line $(\mathrm{P}=0.17)$. Among those given nal-IRI $+5-\mathrm{FU} / \mathrm{LV}$ as $2^{\text {nd }}$ line, use of gemcitabineinclusive chemotherapy as the $1^{\text {st }}$ line agent did not impact survival $(\mathrm{P}=0.68)$. Prior irinotecan exposure and baseline CA 19-9 level did not affect the overall survival (OS) but patients with a higher CA 19-9 level had a significant risk of progression ( $\mathrm{HR}=3.2, \mathrm{P}=0.02$ ). Grade $3 / 4$ toxicities were reported in only $19 \%$ patients.

Conclusions: Our report suggests that nal-IRI + 5-FU/LV offers a modest survival benefit with a tolerable safety profile as an advanced line of treatment in patients with advanced PAC.

Keywords: Liposomal irinotecan; MM-398; nano-liposomal irinotecan (nal-IRI); pancreatic cancer; $2^{\text {nd }}$ line treatment

Submitted Aug 19, 2020. Accepted for publication Jan 31, 2021.

doi: 10.21037/jgo-20-338

View this article at: http://dx.doi.org/10.21037/jgo-20-338

(C) Journal of Gastrointestinal Oncology. All rights reserved. 


\section{Introduction}

Advanced pancreatic adenocarcinoma (PAC) remains an insurmountable challenge and is estimated to cause 47,050 deaths in the US in 2020 with a 5 -year survival rate ranging from a mere $2-9 \%(1-3)$. Within the past decade, combination therapy with nab-paclitaxel plus gemcitabine (Gem-Abraxane) or FOLFIRINOX (folinic acid, 5 -fluorouracil, irinotecan and oxaliplatin) has shown survival benefit as compared to gemcitabine monotherapy, leading to a paradigm shift in the treatment of advanced pancreatic cancer (4-6). Despite the use of newer combination chemotherapeutic agents, overall survival (OS) with $1^{\text {st }}$ line treatment remains less than 1 year on an average (4-8). In phase 3 randomized controlled trials, FOLFIRINOX was shown to significantly increase OS from 6.8 to 11.1 months while Gem-Abraxane demonstrated an OS of $8.5 \mathrm{vs}$. 6.7 months $(\mathrm{P}<0.001)$ when compared to gemcitabine monotherapy, albeit at the cost of increased risk of myelosuppression and neuropathy with both regimens $(5,6)$. Similarly, progression-free survival (PFS) for the recommended $1^{\text {st }}$ line regimens such as gemcitabine, Gem-Abraxane, and FOLFIRINOX are only 3.4, 5.5, and 6.4 months, respectively (5-7). As such, most patients require $2^{\text {nd }}$ line chemotherapy, but options remain scarce. A promising $2^{\text {nd }}$ line combination of nano-liposomal irinotecan (nal-IRI) with 5 -fluorouracil/leucovorin $(5-\mathrm{FU} / \mathrm{LV})$ emerged from the NAPOLI-1 trial, the largest global phase 3 trial to date, testing a $2^{\text {nd }}$ line regimen for patients with metastatic pancreatic cancer (9). Patients were randomly assigned to 3 arms: nal-IRI + 5-FU/LV vs. nal-IRI alone vs. 5-FU/LV alone. The study reported improved OS (6.1 vs. 4.2 months, $\mathrm{P}=0.012)$ and $\mathrm{PFS}(3.1$ vs. 1.5 months, $\mathrm{P}=0.0001)$ with nal-IRI + 5-FU/LV when compared to 5-FU/LV alone. The unique formulation of nal-IRI allows for liposomal delivery of irinotecan within the tumor, resulting in $>5$-fold greater intratumoral accumulation of the active agent as compared to that achieved by free unencapsulated irinotecan resulting in increased efficacy and decreased toxicity (10-12).

Despite the 2015 FDA approval of this combination, there is limited post-approval real-world data regarding its efficacy, safety and optimal sequencing. NAPOLI-1 trial only enrolled patients who failed prior gemcitabine-based therapy, leaving questions about response in patients with prior irinotecan-based therapy unanswered. Therefore, the purpose of this retrospective analysis is to expand on the currently available literature by sharing our institutional experiences regarding the effectiveness and safety of this combination as an advanced line of therapy in patients with advanced PAC irrespective of prior exposures, outside of the controlled environment of a clinical trial.

We present the following article in accordance with the STROBE reporting checklist (available at http://dx.doi. org/10.21037/jgo-20-338).

\section{Methods}

\section{Participants and setting}

We designed an observational study involving retrospective analysis of the electronic medical records of patients with advanced PAC (locally advanced unresectable or metastatic disease) who were given nal-IRI and 5-FU/LV following failure of one or more lines of therapy. Patients in this study were treated with nal-IRI + 5-FU/LV from August 2015 to December 2018 at the Kansas University Medical Center (KUMC) and University of Alabama at Birmingham (UAB). All patients $(\mathrm{n}=58)$ who were administered even a single cycle of nal-IRI + 5-FU/LV during the study period were included to avoid any potential selection bias. All patients were followed until December 31 $1^{\text {st }}, 2019$ (cut-off date).

\section{Data collection}

Data regarding age, gender, race, functional status [Eastern Cooperative Oncology Group (ECOG) performance status score], baseline CA 19-9 levels, details of prior lines of treatment, specifically prior treatment with irinotecan or Gem-Abraxane was extracted from the electronic medical record. Other variables collected include date of initiation and initial dose strength of nal-IRI + 5-FU/LV, number of dose modifications, dose delays, treatment related side effects, date of progression and date of death.

\section{Outcome measures}

The main outcomes of interest in this analysis were effectiveness and tolerability of nal-IRI +5 -FU/LV in cases of advanced PAC after failure of initial lines of therapy. Efficacy of the regimen was defined in terms of OS and PFS. Tolerability of the regimen was determined by the incidence of treatment-associated side effects which were defined in accordance with the National Cancer Institute 
Table 1 Baseline patient characteristics

\begin{tabular}{|c|c|}
\hline Characteristics & Total enrolled $(n=58)$ \\
\hline Age (years), median [range] & 65.5 [38-82] \\
\hline \multicolumn{2}{|l|}{ Gender, n (\%) } \\
\hline Male & $30(51.72)$ \\
\hline Female & $28(48.28)$ \\
\hline \multicolumn{2}{|l|}{ Race, n (\%) } \\
\hline White & $47(81.03)$ \\
\hline Black & $10(17.24)$ \\
\hline Other & $1(1.72)$ \\
\hline \multicolumn{2}{|l|}{ ECOG, n (\%) } \\
\hline 0 & $10(17.24)$ \\
\hline 1 & $44(75.86)$ \\
\hline 2 & $3(5.17)$ \\
\hline 3 & $1(1.72)$ \\
\hline \multicolumn{2}{|l|}{ Prior lines of treatment, $n(\%)$} \\
\hline 1 & $31(53.45)$ \\
\hline 2 & $18(31.03)$ \\
\hline 3 & $9(15.52)$ \\
\hline \multicolumn{2}{|l|}{ CA 19-9 levels, n (\%) } \\
\hline High (>200 U/mL) & $30(51.72)$ \\
\hline Low (200 U/mL) & $28(48.28)$ \\
\hline \multicolumn{2}{|l|}{ Prior irinotecan, n (\%) } \\
\hline Yes & $27(46.55)$ \\
\hline No & $31(53.45)$ \\
\hline \multicolumn{2}{|c|}{ Among patients receiving nal-IRI as $2^{\text {nd }}$ line, $\mathrm{n}(\%)$} \\
\hline \multicolumn{2}{|c|}{$\begin{array}{l}\text { Prior gemcitabine-based treatment } \\
\text { in } 1^{\text {st }} \text { line }\end{array}$} \\
\hline Yes & $24(77.42)$ \\
\hline No & $7(22.58)$ \\
\hline
\end{tabular}

ECOG, Eastern Cooperative Oncology Group; nal-IRI, nanoliposomal irinotecan.

Common Terminology Criteria for Adverse Events (NCI-CTCAE V4.0). Exposures of interest also included factors that may have impacted OS; such as prior lines of chemotherapy, previous exposure to irinotecan, prior $1^{\text {st }}$ line therapy with Gem-Abraxane, baseline CA 19-9 levels and ECOG status. Response to nal-IRI + 5-FU/LV was determined by examining periodic CT scans (every 8-12 weeks) and using the RECIST Criteria 1.1 (13).

\section{Statistical analysis}

PFS is computed since the initial date of receiving nal-IRI +5 -FU/LV to progression of disease or demise, whatever came first. OS is computed since the initial date of receiving nal-IRI + 5-FU/LV to death. Disease control rate (DCR) is computed by dividing the number of cases who achieved either complete response (CR), partial response (PR) or stable disease (SD) by the total of number of cases. KaplanMeier curves for OS, PFS and differences in survival across covariates such as prior irinotecan exposure and prior gemcitabine-based treatment in those receiving nal-IRI + $5-\mathrm{FU} / \mathrm{LV}$ in $2^{\text {nd }}$ line setting were created using log-rank test. Using Cox proportional hazard regression analysis, the strength of association between different covariates and risk of mortality or progression were reported. Quantitative variables such as CA 19-9 level and starting dose of nal-IRI were grouped as high $v s$. low (with a cut-off of $200 \mathrm{U} / \mathrm{mL}$ ) and $70 \mathrm{mg} / \mathrm{m}^{2}$ vs. other doses respectively; and analyzed as categorical variables. There were no missing data or loss to follow-up during the study period.

\section{Ethical statement}

The study was conducted in accordance with the Declaration of Helsinki (as revised in 2013). Research and individual consent waiver were granted due to the retrospective nature of the study, and the study was approved by the Institutional Review Board (STUDY000003970).

\section{Results}

\section{Patient characteristics}

From August 2015 to December 2018, 58 patients were administered, at the least, a single cycle of nal-IRI + 5-FU/ LV. Baseline characteristics are enlisted in Table 1.

The median age of the cohort was 65.5 years. The majority (93\%) had an ECOG score of 0 or $1.70 .6 \%$ had metastatic PAC and the rest (29.4\%) had locally advanced unresectable PAC. All patients had been previously treated. Half of the study population only had one prior line of treatment. $46.5 \%$ of the patients had received irinotecan as a prior line of therapy. Among patients receiving nal-IRI + 


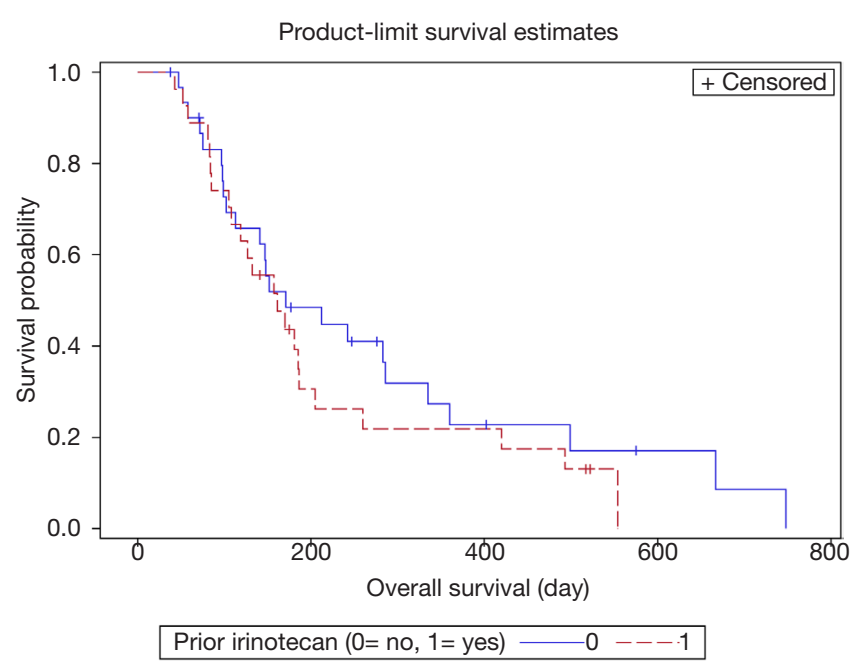

Figure 1 OS for patients with pancreatic cancer treated with nal$\mathrm{IRI}+5-\mathrm{FU} / \mathrm{LV}$ and stratified based on history of prior irinotecan treatment. The difference in the $\mathrm{OS}$ is not significant $(\mathrm{P}$ value =0.3726). OS, overall survival; nal-IRI, nano-liposomal irinotecan; 5-FU/LV, 5-fluorouracil/leucovorin.

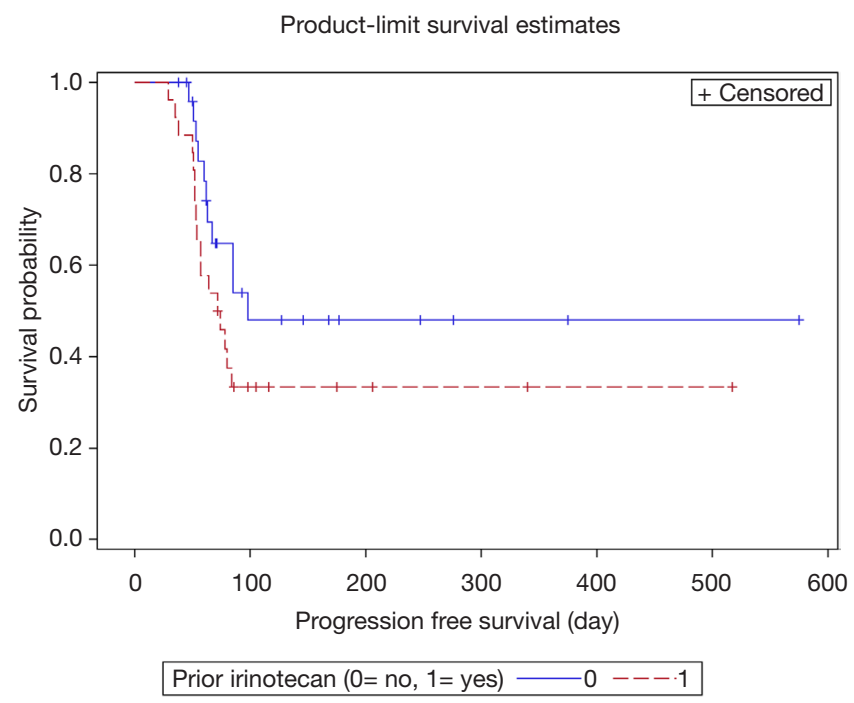

Figure 2 PFS for patients with pancreatic cancer treated with nalIRI $+5-F U / L V$ and stratified based on history of prior irinotecan treatment. The difference in the PFS is not significant ( $\mathrm{P}$ value $=0.0590$ ). PFS, progression-free survival; nal-IRI, nano-liposomal irinotecan; 5-FU/LV, 5-fluorouracil/leucovorin.

5-FU/LV directly after $1^{\text {st }}$ line, $77 \%$ (24/31) were previously treated with gemcitabine-based regimen as the $1^{\text {st }}$ line therapy. Only about half the study population had a high
CA 19-9 level. None of the patients had missing data or any variable of interest.

\section{Dosing schedule and reductions}

Doses ranged from $50-70 \mathrm{mg} / \mathrm{m}^{2}$. Majority of the patients (67\%) initiated treatment with the standard $70 \mathrm{mg} / \mathrm{m}^{2}$ dose of nal-IRI. About $26 \%$ received a lower starting dose of $50 \mathrm{mg} / \mathrm{m}^{2}$ as per physician discretion based on tolerance to prior therapy and functional status. Seven patients (12\%) required 1 dose reduction while only 4 patients (7\%) needed 2 dose reductions in the study. Twenty-four (41\%) patients had at least one or more dose delays. However, these dose reductions and delays did not affect overall outcomes. Fatigue, anemia and diarrhea were the frequent causes of dose reductions. The cohort was administered a median of 4 doses of nal-IRI until the cut-off date. Among those given nal-IRI as a $2^{\text {nd }}$ line agent, gemcitabine alone and Gem-Abraxane were the $1^{\text {st }}$ line therapies in $6(20.6 \%)$ and $18(62 \%)$ patients, respectively; 4 patients $(13.7 \%)$ received FOLFIRINOX as the initial therapy whereas one patient was treated with gemcitabine-cisplatin combination. Those given nal-IRI beyond $2^{\text {nd }}$ line had been exposed to variable sequences of gemcitabine or fluoropyrimidine based combination therapies.

\section{Efficacy}

The median OS was 5.4 months for our study sample with a median follow up of 6 months. Patients were stratified into those with or without prior irinotecan exposure while patients given nal-IRI $+5-\mathrm{FU} / \mathrm{LV}$ as $2^{\text {nd }}$ line were stratified into those who had received a gemcitabine-inclusive combination as $1^{\text {st }}$ line versus others. There were no statistically significant differences in the PFS and OS among these subgroups (Figures 1,2, Figures S1,S2) although patients without prior irinotecan exposure trended towards an improved PFS (P value $=0.059$ ). Majority of patients had SD $(41.38 \%)$, with $2(3.45 \%)$ and $4(6.9 \%)$ patients experiencing complete remission and partial remission as per the RECIST 1.1 criteria, respectively (Table 2).

Disease progression was reported in 28 patients (48\%). The overall DCR was $51.72 \%$. DCR was higher for the subgroup given nal-IRI +5 -FU/LV directly post$1^{\text {st }}$ line treatment $(63.33 \%)$ as compared to beyond $2^{\text {nd }}$ line $(36.67 \%)$. Among those given nal-IRI + 5-FU/LV immediately post- $1^{\text {st }}$ line, DCR was even better for the 
Table 2 Treatment and outcomes of patients treated with nal-IRI + 5-FU/LV

\begin{tabular}{|c|c|}
\hline & $N(\%)$ \\
\hline \multicolumn{2}{|l|}{ Nal-IRI administration, $\mathrm{n}(\%)$} \\
\hline $2^{\text {nd }}$ line & $31(53.45)$ \\
\hline$>2^{\text {nd }}$ line & $27(46.55)$ \\
\hline \multicolumn{2}{|c|}{ Starting dose of nal-IRI $\left(\mathrm{mg} / \mathrm{m}^{2}\right), \mathrm{n}(\%)$} \\
\hline 50 & $15(25.86)$ \\
\hline 55 & $1(1.72)$ \\
\hline 60 & $2(3.45)$ \\
\hline 65 & $1(1.72)$ \\
\hline 70 & $39(67.24)$ \\
\hline \multicolumn{2}{|l|}{ Response, n (\%) } \\
\hline $\mathrm{CR}$ & $2(3.45)$ \\
\hline PR & $4(6.90)$ \\
\hline SD & $24(41.38)$ \\
\hline PD & $28(48.28)$ \\
\hline \multicolumn{2}{|l|}{ Patients died at cut-off date, n (\%) } \\
\hline Yes & 47 (81.03) \\
\hline No & $11(18.97)$ \\
\hline OS (days), median (95\% Cl) & $161(127-212)$ \\
\hline PFS (days), median (95\% Cl) & 80 (60-no upper limit) \\
\hline \multicolumn{2}{|l|}{$\mathrm{DCR}[(\mathrm{CR}+\mathrm{PR}+\mathrm{SD}) / \mathrm{n}], \%$} \\
\hline Prior irinotecan & 37.04 \\
\hline Prior Gem-Abraxane & 67.86 \\
\hline Nal-IRI administered as $2^{\text {nd }}$ line & 61.29 \\
\hline
\end{tabular}

nal-IRI, nano-liposomal irinotecan; 5-FU/LV, 5-fluorouracil/ leucovorin; OS, overall survival; PFS, progression-free survival; $\mathrm{DCR}$, disease control rate; $\mathrm{CR}$, complete response; PR, partial response; $\mathrm{SD}$, stable disease; $\mathrm{PD}$, progressive disease.

subset that received gemcitabine-based treatment in $1^{\text {st }}$ line therapy versus those who did not $(84.21 \%$ vs. $15.79 \%)$. Baseline CA 19-9 level was not associated with risk of mortality but significantly increased the odds of progression in those with elevated CA 19-9 levels ( $\mathrm{HR}=3.27$; 95\% CI $=1.17,9.15 ; \mathrm{P}=0.02)$ compared to those with normal CA 19-9 levels. All the patient and treatment related factors analyzed for association with PFS or OS are enlisted in Table 3.
Majority of patients (53\%) were given nal-IRI + 5-FU/ $\mathrm{LV}$ directly following failure of $1^{\text {st }}$ line therapy. There was no significant difference in OS and PFS between patients who received nal-IRI in $2^{\text {nd }}$ line and those receiving it beyond $2^{\text {nd }}$ line (Figures S3,S4). Twenty-two percent of those who progressed after nal-IRI + 5-LU/LV went on to clinical trials, 24 percent received FOLFOX and the rest were transitioned to hospice.

\section{Adverse events}

The most frequent treatment-related adverse effects (AEs) included fatigue and anemia. Although the incidence of fatigue and anemia is high, $69 \%$ and $65 \%$ of these, respectively, were classified as grade 1 in severity. There was only one incidence of grade 4 neutropenia. Other serious side effects were limited to grade 3 toxicity and were relatively few. No drug related mortality or drug discontinuation was reported. All side effects along with grades are listed in Table 4.

\section{Discussion}

Nearly a decade ago, most patients with advanced PAC were managed with best supportive care (BSC) after progressing on initial therapy. A systematic meta-analysis of 34 clinical studies suggested a survival benefit with $2^{\text {nd }}$ line treatment over BSC alone (median OS of 6.0 vs. 2.8 months, $\mathrm{P}=0.01$ ) (14). In 2015, the National Cancer Care Network (NCCN) guidelines adapted nal-IRI + 5-FU/LV as a $2^{\text {nd }}$ line therapy, post-failure of gemcitabine-inclusive regimens for advanced PAC, based on NAPOLI-1 trial (9). Our analysis is one of the few post-approval, real-world, clinical outcomes assessment with nal-IRI + 5-FU/LV and showed survival benefits similar to that reported in the NAPOL-1 trial with a manageable safety profile. To evaluate the appropriate sequencing for various regimens, we also categorized patients based on prior irinotecan and prior Gem-Abraxane exposure and analyzed their impact on response to nal-IRI + 5-FU/LV.

Our population is comparable to that of the NAPOLI-1 trial in terms of gender distribution, performance status and percentage of patients given nal-IRI $+5-\mathrm{FU} / \mathrm{LV}$ as $2^{\text {nd }}$ line agent (53\% in both studies) (9). However, our cohort includes a significantly higher percentage of patients who were previously exposed to irinotecan $(46.5 \%$ vs. $10 \%)$. We 
Table 3 Factors predicting OS and PFS in patients treated with nal-IRI + 5-FU/LV

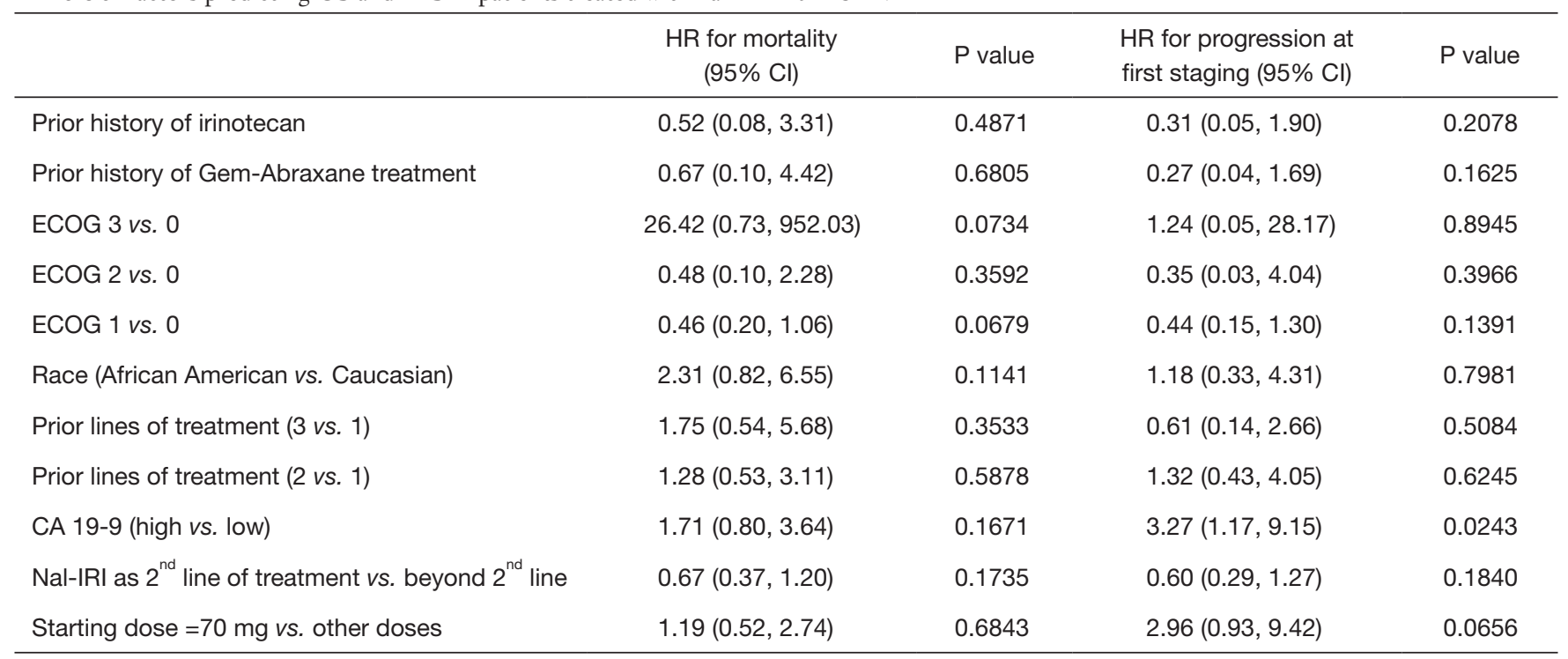

OS, overall survival; PFS, progression-free survival; nal-IRI, nano-liposomal irinotecan; 5-FU/LV, 5-fluorouracil/leucovorin; ECOG, Eastern Cooperative Oncology Group.

Table 4 Treatment-related adverse events

\begin{tabular}{lcc}
\hline Side effect (total $\mathrm{n}=58)$ & Any grade, $\mathrm{n}(\%)$ & Grade 3/4, $\mathrm{n}(\%)$ \\
\hline Fatigue & $52(89.7)$ & $1(1.7)$ \\
Diarrhea & $24(41.4)$ & 0 \\
Nausea/vomiting & $15(25.9)$ & 0 \\
Anemia & $49(84.5)$ & $7(12.1)$ \\
Neutropenia & $15(25.9)$ & $1(1.7)$ \\
Thrombocytopenia & $24(41.4)$ & $1(1.7)$ \\
Neuropathy & $19(32.8)$ & 0 \\
Elevated creatinine & $9(15.5)$ & $1(1.7)$ \\
Transaminitis & $12(20.7)$ & 0 \\
\hline
\end{tabular}

share similarity with the cohort reported by Glassman et al. in this regard where $59 \%$ patients were previously treated with an irinotecan-based regimen (15). They reported a reduced OS and PFS for patients who failed prior irinotecan-based regimens. This finding was attributed in part to the fact that those who progressed after irinotecancombination regimen were given nal-IRI + 5-FU/LV later in the disease course i.e., median of $3^{\text {rd }}$ line (15). Concerns for resistance to nal-IRI in those previously exposed to irinotecan have also been raised by two recent real-world studies from Korea and US, both demonstrating enhanced survival in patients with no history of irinotecan use $(16,17)$. Contrary to this, we found no significant difference in the OS on subgroup analyses between those with or without prior exposure to irinotecan. However, irinotecannaïve patients tended towards a better PFS ( $\mathrm{P}$ value $=0.059)$ although it did not reach statistical significance owing to a small sample size. The NCCN guidelines currently recommend using nal-IRI $+5-\mathrm{FU} / \mathrm{LV}$ post fluoropyrimidine-inclusive therapy only if there is no prior exposure to irinotecan (category 1 recommendation) (18). Although the DCR was better for those receiving nal-IRI +5 -FU/LV directly after $1^{\text {st }}$ line versus beyond $2^{\text {nd }}$ line (63.3\% vs. $36.7 \%$ ) in our cohort, this did not translate into a significantly improved PFS or OS owing to a small sample size. A trend in the improvement of OS and PFS was observed if nal-IRI + 5-FU/LV was given earlier in the disease course $(15,17,19)$.

Glassman et al. reported improved PFS and OS when treated with gemcitabine alone or Gem-Abraxane followed by nal-IRI + 5-FU/LV compared to receiving this in later lines of therapy (15). To assess if the line of therapy is a confounder, we categorized patients treated with nal-IRI + 5 -FU/LV in $2^{\text {nd }}$ line into two groups; based on the $1^{\text {st }}$ line therapy received as gemcitabine-inclusive regimen versus non-gemcitabine-based regimens. There was no difference in PFS and OS, however the DCR was higher for those 
pre-treated with gemcitabine-based therapies (84.21\% vs. $15.79 \%)$. Kieler et al have previously demonstrated a survival benefit in patients with a higher CA 19-9 who receive nal-IRI + 5-FU/LV as opposed to oxaliplatin + 5-FU combination, suggesting a potential role for CA 19-9 as a biomarker for choosing a nal-IRI based regimen (19). The higher baseline CA 19-9 levels did not increase the risk of mortality in our study.

The median OS (5.4 months) reported in our study is comparable to the NAPOLI- 1 trial (median OS $=6.1$ months) as well as other similar real-world studies by Glassman et al. (median OS $=5.3$ months) and Kieler $e$ al . (median OS $=6.7$ months) $(9,15,19)$. Two recent studies conducted in Korean and Taiwanese cohorts have also shown similarly promising real-world survival outcomes (median OS =9.4 and 6.6 months, respectively) $(16,20)$.

As per the NCCN guidelines, additional options for $2^{\text {nd }}$ line use in locally advanced or metastatic PAC include irinotecan-based regimens such as FOLFIRI, FOLFIRINOX or capecitabine with and without oxaliplatin, the OFF regimen (oxaliplatin, folinic acid and 5-fluorouracil) and mFOLFOX-6 (18,21). The OFF regimen demonstrated significantly higher OS and PFS as opposed to 5-FU/LV alone in the CONKO-003 trial (22). However, the subsequent PANCREOX trial yielded discordant results better survival outcomes with 5-FU/ LV (23). Hitherto, there have been no randomized phase 3 trials evaluating these as an advanced line of therapy. An Italian phase 2 trial demonstrated a modest response to FOLFIRI (median OS $=5$ months) albeit with a high toxicity (55\% rate of grade $3 / 4 \mathrm{AEs}$ ) (24). A retrospective series looking at 63 patients receiving FOLFIRI post progression of disease on 1-3 lines of gemcitabine \pm platinum based regimens reported an encouraging median OS of 6.6 months (25). Role of FOLFIRINOX postgemcitabine failure has been examined in a small phase 2 study by Kobayashi et al. with a promising median OS of 9.8 months although a high rate of neutropenia (66.7\%) was noted (26). Xelox i.e., capecitabine and oxaliplatin for gemcitabine resistant disease was examined in two phase 2 studies resulting in similar outcomes with a median OS of 5.7 and 5.3 , respectively $(27,28)$. The survival statistics reported by the aforementioned trials evaluating various regimens seem similar to nal-IRI + 5-FU/LV on face value, however, evidence is limited and stems from underpowered phase 2 or retrospective studies and no head to head comparisons exist. Having said that, a 2017 meta-analysis revealed that irinotecan and 5-FU combinations postgemcitabine failure may confer a greater survival advantage as opposed to $5-\mathrm{FU}+$ oxaliplatin regimens or $5-\mathrm{FU}$ alone although both appeared to improve PFS (29). Kieler et al. also demonstrated a better OS and PFS in a matched cohort of patients given nal-IRI + 5-FU/LV versus oxaliplatin plus 5-FU combination for advanced PAC (median OS 9.33 vs. 6.18 months, $\mathrm{P}=0.03$ ) (19). These studies suggest that oxaliplatin based combinations might be less beneficial in advanced settings and randomized clinical trials to prove these findings are needed.

In terms of the safety profile, the NAPOLI 1 trial reported neutropenia (27\%), fatigue (14\%), diarrhea (13\%), vomiting $(11 \%)$, and anemia (9\%) to be the most common grade 3 and 4 treatment-related adverse events (9). Our study cohort reported anemia (12\%), neutropenia $(<2 \%)$, thrombocytopenia $(<2 \%)$, and fatigue $(<2 \%)$ to be the most frequent grade $\geq 3 \mathrm{AEs}$. The relatively low proportion of serious AEs can be attributed to a one-third of the patients receiving a lower initial dose in our study. Glassman et al. also reported lower frequencies of grade 3/4 AEs i.e., anemia (18\%), nausea (4\%), vomiting (4\%), fatigue (2\%), diarrhea (2\%) and neutropenia (2\%), however $70 \%$ patients in their cohort were treated with lower than recommended dose of $70 \mathrm{mg} / \mathrm{m}^{2}$ (15). Dose reductions were not too common in our study and we did not find any significant association between dose reduction and worse outcomes. These findings are comparable to the results of the previous studies $(9,15,19)$. In fact, Glassman et al. depicted a positive impact of dose reductions on survival measures (15). Since the NAPOLI-1 trial found no difference in the quality of life (QoL) among the cohorts despite prolonging survival, this comes as an important finding and supports the notion that dose adjustments to improve drug tolerability can empower patients to have a better QoL without compromising the overall drug efficacy. Besides, our drug dosing strategy better mimics actual practice as evidenced by the recent large US database study indicating that $44.5 \%$ patients received a lesser than standard nal-IRI dosage (30$\left.65 \mathrm{mg} / \mathrm{m}^{2}\right)$ in clinical practice (17).

Our study is limited by a retrospective design with a small sample size, which may have led to some falsenegative results due to lack of statistical power. However, our study could be more generalizable as it took place across two tertiary level treatment centers in the Midwest and South-Eastern USA, compared to single center studies by Glassman et al. and Kieler et al. $(15,19)$ Also, to avoid any 
potential selection bias, all patients who received even one cycle of nal-IRI + 5-FU/LV were included in the analysis. Our findings support the clinical effectiveness of nal-IRI $+5-\mathrm{FU} / \mathrm{LV}$ as an advanced line of therapy post failure of gemcitabine alone or gemcitabine-inclusive combinations and probes into its potential role after irinotecan-based therapies. Future studies may need to investigate the biomarkers in predicting response to nal-IRI.

\section{Conclusions}

This report is among the few actual clinical effectiveness and safety analyses of nal-IRI +5 -FU/LV conducted across two cancer centers in the US in a real-world clinical setting. The survival outcome reported in our study is modest (median OS $=5.4$ months) but encouraging for patients with advanced PAC and limited treatment options. Importantly, we did not find any difference in outcomes in patients given nal-IRI +5 -FU/LV earlier or later $\left(>2^{\text {nd }}\right.$ lines $)$ in the disease course as well as those with or without prior irinotecan exposure. These results call for further validation in randomized controlled trials to broaden the horizon for future clinical applications. Furthermore, our cohort experienced a relatively low incidence of treatment-related serious AEs. This is of utmost priority for patients with aggressive PAC who are debilitated from the underlying disease and any additional therapy-related toxicity can have a drastic detrimental effect on their QoL.

\section{Acknowledgments}

Funding: None.

\section{Footnote}

Reporting Checklist: The authors have completed the STROBE reporting checklist. Available at http://dx.doi. org/10.21037/jgo-20-338

Data Sharing Statement: Available at http://dx.doi. org/10.21037/jgo-20-338

Peer Review File: Available at http://dx.doi.org/10.21037/ jgo-20-338

Conflicts of Interest: All authors have completed the ICMJE uniform disclosure form (available at http://dx.doi. org/10.21037/jgo-20-338). Dr. RP is a consultant for Ipsen pharmaceuticals. This has no influence on the data and presentation of this study. No personnel from Ipsen involved or associated with this study either directly or indirectly. The other authors have no conflicts of interest to declare.

Ethical Statement: The authors are accountable for all aspects of the work in ensuring that questions related to the accuracy or integrity of any part of the work are appropriately investigated and resolved. The study was conducted in accordance with the Declaration of Helsinki (as revised in 2013). Research and individual consent waiver were granted due to the retrospective nature of the study, and the study was approved by the Institutional Review Board (STUDY000003970).

Open Access Statement: This is an Open Access article distributed in accordance with the Creative Commons Attribution-NonCommercial-NoDerivs 4.0 International License (CC BY-NC-ND 4.0), which permits the noncommercial replication and distribution of the article with the strict proviso that no changes or edits are made and the original work is properly cited (including links to both the formal publication through the relevant DOI and the license). See: https://creativecommons.org/licenses/by-nc-nd/4.0/.

\section{References}

1. Siegel RL, Miller KD, Jemal A. Cancer statistics, 2020. CA Cancer J Clin 2020;70:7-30.

2. Howlader N, Noone AM, Krapcho M, et al. SEER Cancer Statistics Review, 1975-2016. Bethesda: National Cancer Institute. Available online: https://seer.cancer.gov/ csr/1975_2016/ (Accessed on April 30, 2019).

3. McGuigan A, Kelly P, Turkington RC, et al. Pancreatic cancer: A review of clinical diagnosis, epidemiology, treatment and outcomes. World J Gastroenterol 2018;24:4846-61.

4. Von Hoff DD, Ervin TJ, Arena FP, et al. Results of a randomized phase III trial (MPACT) of weekly nabpaclitaxel plus gemcitabine versus gemcitabine alone for patients with metastatic adenocarcinoma of the pancreas with PET and CA19-9 correlates. Am Soc Clin Oncol 2013;369:1691-703.

5. Von Hoff DD, Ervin T, Arena FP, et al. Increased survival in pancreatic cancer with nab-paclitaxel plus gemcitabine. 
N Engl J Med 2013;369:1691-703.

6. Conroy T, Desseigne F, Ychou M, et al. FOLFIRINOX versus gemcitabine for metastatic pancreatic cancer. $\mathrm{N}$ Engl J Med 2011;364:1817-25.

7. Burris HA III, Moore MJ, Andersen J, et al. Improvements in survival and clinical benefit with gemcitabine as firstline therapy for patients with advanced pancreas cancer: a randomized trial. J Clin Oncol 1997;15:2403-13.

8. Moore MJ, Goldstein D, Hamm J, et al. Erlotinib plus gemcitabine compared with gemcitabine alone in patients with advanced pancreatic cancer: a phase III trial of the National Cancer Institute of Canada Clinical Trials Group. J Clin Oncol 2007;25:1960-6.

9. Wang-Gillam A, Li CP, Bodoky G, et al. Nanoliposomal irinotecan with fluorouracil and folinic acid in metastatic pancreatic cancer after previous gemcitabine-based therapy (NAPOLI-1): a global, randomised, open-label, phase 3 trial. Lancet 2016;387:545-57.

10. Drummond DC, Noble CO, Guo Z, et al. Development of a highly active nanoliposomal irinotecan using a novel intraliposomal stabilization strategy. Cancer Res 2006;66:3271-7.

11. Adiwijaya BS, Kim J, Lang I, et al. Population Pharmacokinetics of Liposomal Irinotecan in Patients with Cancer. Clin Pharmacol Ther 2017;102:997-1005.

12. Chiang NJ, Chang JY, Shan YS, et al. Development of nanoliposomal irinotecan (nal-IRI, MM-398, PEP02) in the management of metastatic pancreatic cancer. Expert Opin Pharmacother 2016;17:1413-20.

13. Eisenhauer EA, Therasse P, Bogaerts J, et al. New response evaluation criteria in solid tumors: revised RECIST guideline (version 1.1). Eur J Cancer 2009;45:228-47.

14. Rahma OE, Duffy A, Liewehr DJ, et al. Second-line treatment in advanced pancreatic cancer: a comprehensive analysis of published clinical trials. Ann Oncol 2013;24:1972-9.

15. Glassman DC, Palmaira RL, Covington CM. Nanoliposomal irinotecan with fluorouracil for the treatment of advanced pancreatic cancer, a single institution experience. BMC Cancer 2018;18:693.

16. Yoo C, Im HS, Kim KP, et al. Real-world efficacy and safety of liposomal irinotecan plus fluorouracil/leucovorin in patients with metastatic pancreatic adenocarcinoma: a study by the Korean Cancer Study Group. Ther Adv Med Oncol 2019;11:1758835919871126.

17. Barzi A, Miksad R, Surinach A, et al. Real-world dosing patterns and outcomes of patients with metastatic pancreatic cancer treated with a liposomal irinotecan regimen in the United States. Pancreas 2020;49:193-200.

18. NCCN Clinical Practice Guidelines in Oncology. Pancreatic Adenocarcinoma. Version 1.2021, October 23, 2021. Available online: https://www.nccn.org/ professionals/physician_gls/pdf/pancreatic.pdf (Accessed on December 21, 2020).

19. Kieler M, Unseld M, Bianconi D, et al. A real-world analysis of second-line treatment options in pancreatic cancer: liposomal-irinotecan plus 5-fluorouracil and folinic acid. Ther Adv Med Oncol 2019;11:1758835919853196.

20. Su YY, Chiang NJ, Tsai HJ, et al. The impact of liposomal irinotecan on the treatment of advanced pancreatic adenocarcinoma: real-world experience in a Taiwanese cohort. Sci Rep 2020;10:7420.

21. PDQ® Adult Treatment Editorial Board. PDQ Pancreatic Cancer Treatment. Bethesda: National Cancer Institute. Updated 07/15/2019. Available online: https://www. cancer.gov/types/pancreatic/hp/pancreatic-treatment-pdq (Accessed on August 31, 2019).

22. Oettle H, Riess H, Stieler JM, et al. Second line oxaliplatin, folinic acid, and fluorouracil versus folinic acid and fluorouracil alone for gemcitabine-refractory pancreatic cancer: outcomes from the CONKO-003 trial. J Clin Oncol 2014;32:2423-9.

23. Gill S, Ko YJ, Cripps C, et al. PANCREOX: a randomized phase III study of fluorouracil/Leucovorin with or without Oxaliplatin for second-line advanced pancreatic Cancer in patients who have received gemcitabine-based chemotherapy. J Clin Oncol 2016;34:3914-20.

24. Zaniboni A, Aitini E, Barni S, et al. FOLFIRI as secondline chemotherapy for advanced pancreatic cancer: a GISCAD multicenter phase II study. Cancer Chemother Pharmacol 2012;69:1641-5.

25. Neuzillet C, Hentic O, Rousseau B, et al. FOLFIRI regimen in metastatic pancreatic adenocarcinoma resistant to gemcitabine and platinum-salts. World J Gastroenterol 2012;18:4533-41.

26. Kobayashi N, Shimamura T, Tokuhisa M, et al. Effect of FOLFIRINOX as second-line chemotherapy for metastatic pancreatic cancer after gemcitabine-based chemotherapy failure. Medicine (Baltimore) 2017;96:e6769.

27. Bayoglu IV, Varol U, Yildiz I, et al. Second line capecitabine and oxaliplatin combination for gemcitabineresistant advanced pancreatic cancer. Asian Pac J Cancer Prev 2014;15:7119-23.

28. Chung KH, Ryu JK, Son JH, et al. Efficacy of capecitabine 
plus oxaliplatin combination chemotherapy for advanced pancreatic cancer after failure of first-line gemcitabinebased therapy. Gut Liver 2017;11:298-305.

29. Vogel A, Ciardiello F, Hubner RA, et al. Post-gemcitabine

Cite this article as: Kasi A, McGinnis T, Naik G, Handa S, Williams G, Paluri R. Efficacy and tolerability of the combination of nano-liposomal irinotecan and 5-fluorouracil/ leucovorin in advanced pancreatic adenocarcinoma: postapproval clinic experience. J Gastrointest Oncol 2021;12(2):464473. doi: 10.21037/jgo-20-338 therapy for patients with advanced pancreatic cancer a comparative review of randomized trials evaluating oxaliplatin- and/or irinotecan-containing regimens. Cancer Treat Rev 2016;50:142-7. 


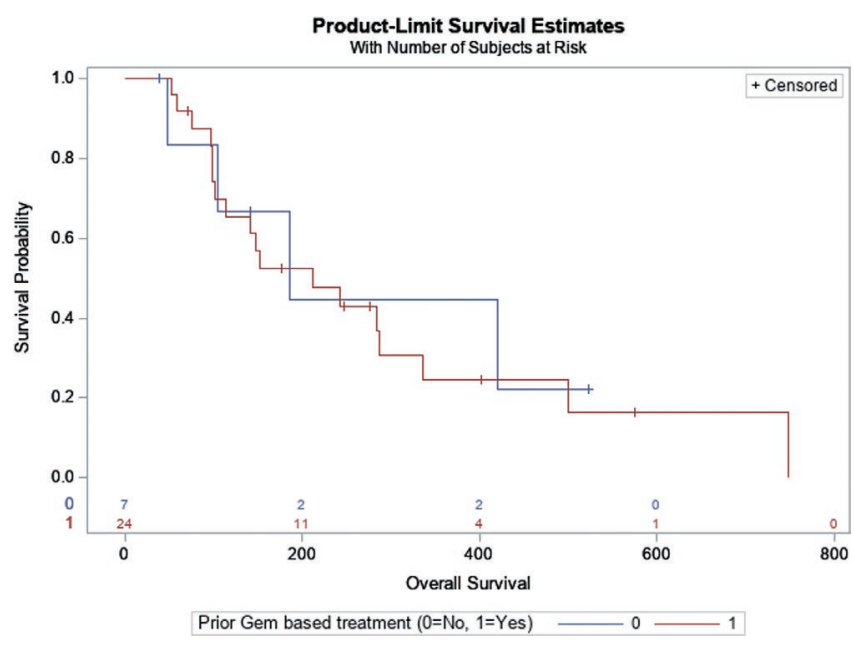

Figure S1 OS for patients with pancreatic cancer treated with nal-IRI $+5-F U / L V$ in $2^{\text {nd }}$ line setting and after stratification based on history of prior gemcitabine-based treatment in 1st line. The difference in OS is not significant ( $\mathrm{P}$ value $=0.7684$ ). OS, overall survival; nal-IRI, nano-liposomal irinotecan; 5-FU/LV, 5-fluorouracil/leucovorin.

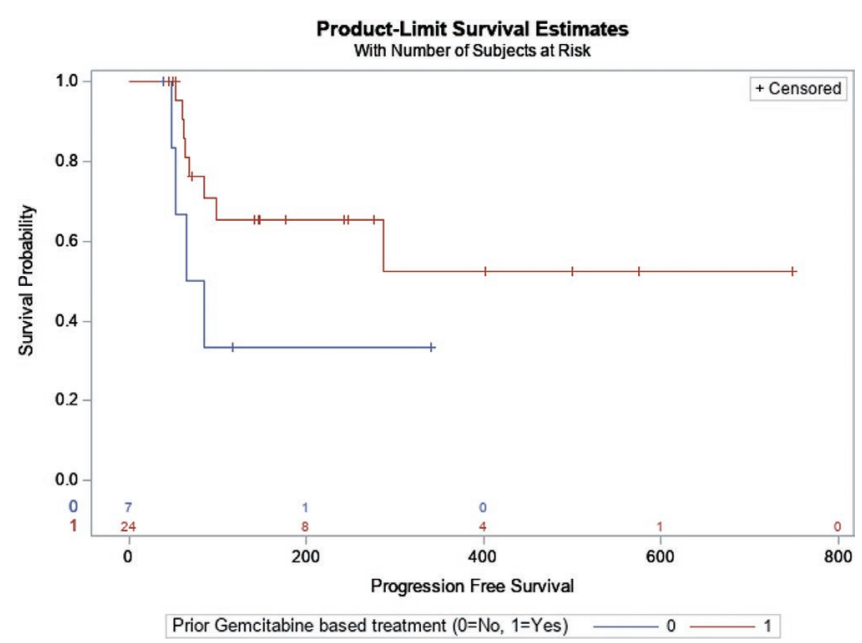

Figure S2 PFS for patients with pancreatic cancer treated with nal-IRI + 5-FU/LV in ${ }^{2 n d}$ line setting and after stratification based on history of prior gemcitabine-based treatment in 1st line setting. The difference in PFS is not significant ( $\mathrm{P}$ value $=0.1341)$. PFS, progression-free survival; nal-IRI, nano-liposomal irinotecan; 5-FU/LV, 5-fluorouracil/leucovorin.

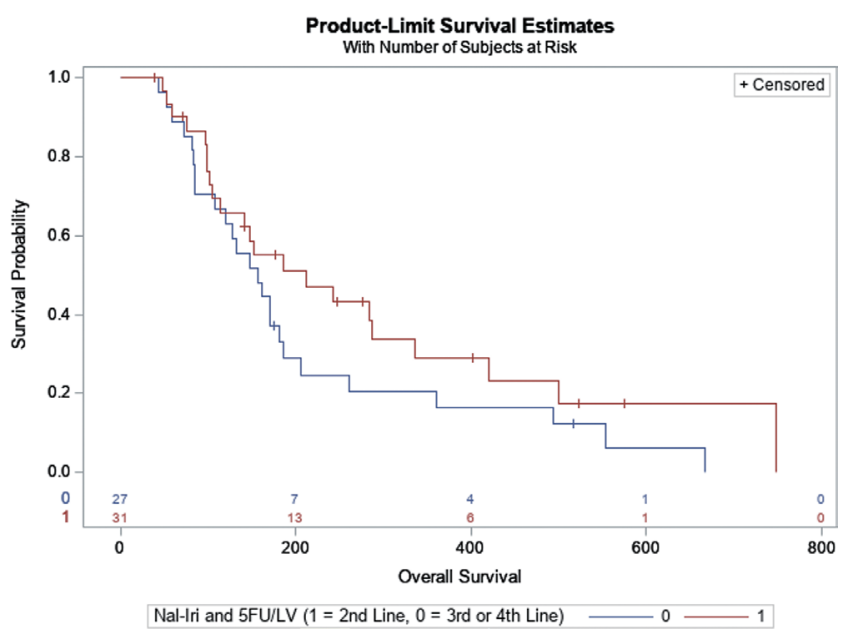

Figure S3 OS of patients with pancreatic cancer treated with nalIRI $+5-F U / L V$ in $2^{\text {nd }}$ line setting or beyond $2^{\text {nd }}$ line setting. There is no significant difference in OS between the two groups ( $\mathrm{P}$ value $=0.1704$ ). OS, overall survival; nal-IRI, nano-liposomal irinotecan; 5-FU/LV, 5-fluorouracil/leucovorin.

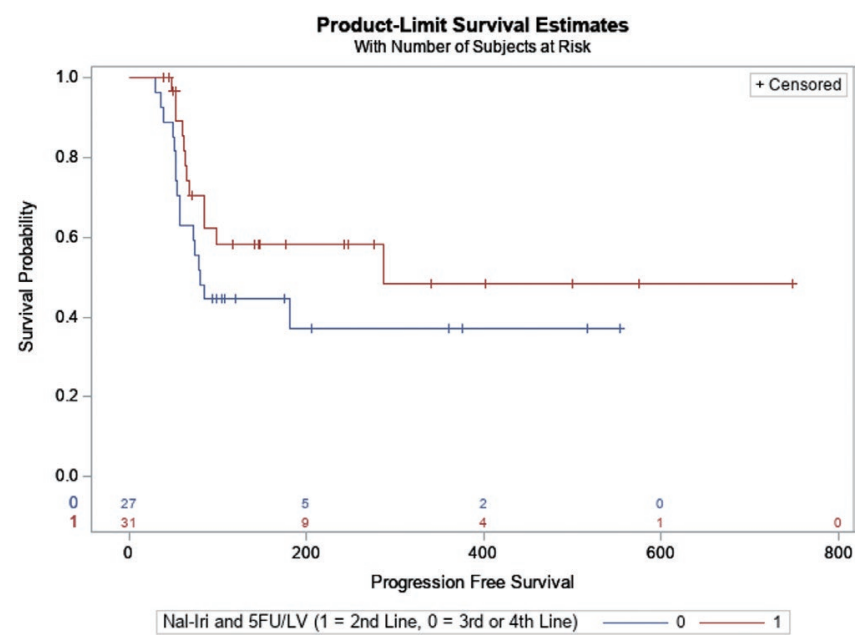

Figure S4 PFS of patients with pancreatic cancer treated with nalIRI $+5-F U / L V$ in $2^{\text {nd }}$ line setting or beyond $2^{\text {nd }}$ line setting. There is no significant difference in PFS between the two groups ( $\mathrm{P}$ value $=0.1799$ ). PFS, progression-free survival; nal-IRI, nano-liposomal irinotecan; 5-FU/LV, 5 -fluorouracil/leucovorin. 